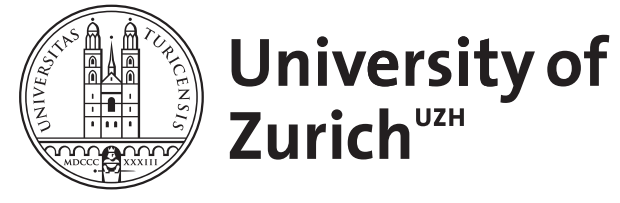

\title{
The Structural Colors of Photonic Glasses
}

\author{
Schertel, Lukas ; Siedentop, Lukas ; Meijer, Janne-Mieke ; Keim, Peter ; Aegerter, Christof M ; Aubry,
} Geoffroy J ; Maret, Georg

\begin{abstract}
The color of materials usually originates from a combination of wavelength-dependent absorption and scattering. Controlling the color without the use of absorbing dyes is of practical interest, not only because of undesired bleaching properties of dyes but also regarding minimization of environmental and health issues. Color control without dyes can be achieved by tuning the material's scattering properties in controlling size and spatial arrangement of scatterers. Herein, calibrated photonic glasses (PGs), which are isotropic materials made by random aggregation of nonabsorbing, monodisperse colloidal polystyrene spheres, are used to generate a wide spectral range of purely structural, angular-independent colors. Experimental reflectance spectra for different sized spheres compare well with a recent theoretical model, which establishes the latter as a tool for color mapping in PGs. It allows to determine the range of visible colors accessible in PGs as function of size, packing fraction, and refractive index of scatterers. It also predicts color saturation on top of the white reflectance as function of the sample's optical thickness. Blue, green, and red are obtained even with low index, while saturated green, cyan, yellow, and magenta can be reached in higher index PGs over several orders of magnitude of sample thickness.
\end{abstract}

DOI: https://doi.org/10.1002/adom.201900442

Posted at the Zurich Open Repository and Archive, University of Zurich

ZORA URL: https://doi.org/10.5167/uzh-172524

Journal Article

Published Version

Originally published at:

Schertel, Lukas; Siedentop, Lukas; Meijer, Janne-Mieke; Keim, Peter; Aegerter, Christof M; Aubry, Geoffroy J; Maret, Georg (2019). The Structural Colors of Photonic Glasses. Advanced Optical Materials, $7(15): 1900442$.

DOI: https://doi.org/10.1002/adom.201900442 


\title{
The Structural Colors of Photonic Glasses
}

\author{
Lukas Schertel,* Lukas Siedentop, Janne-Mieke Meijer, Peter Keim, Christof M. Aegerter, \\ Geoffroy J. Aubry, and Georg Maret*
}

The color of materials usually originates from a combination of wavelengthdependent absorption and scattering. Controlling the color without the use of absorbing dyes is of practical interest, not only because of undesired bleaching properties of dyes but also regarding minimization of environmental and health issues. Color control without dyes can be achieved by tuning the material's scattering properties in controlling size and spatial arrangement of scatterers. Herein, calibrated photonic glasses (PGs), which are isotropic materials made by random aggregation of nonabsorbing, monodisperse colloidal polystyrene spheres, are used to generate a wide spectral range of purely structural, angular-independent colors. Experimental reflectance spectra for different sized spheres compare well with a recent theoretical model, which establishes the latter as a tool for color mapping in PGs. It allows to determine the range of visible colors accessible in PGs as function of size, packing fraction, and refractive index of scatterers. It also predicts color saturation on top of the white reflectance as function of the sample's optical thickness. Blue, green, and red are obtained even with low index, while saturated green, cyan, yellow, and magenta can be reached in higher index PGs over several orders of magnitude of sample thickness. of certain animals and plants. ${ }^{[3-6]}$ Most colors in artificial materials such as paints, cosmetics, and coatings are based on dyes and pigments, i.e., wavelengthdependent light absorbing materials. Similarly, biological colors are often based on light-absorbing proteins called chromophores. However, nature sometimes uses an alternative approach to create most brilliant colors, e.g., in bird feathers or butterfly wings, using wavelength selective scattering. ${ }^{[7-11]}$ Their colors arise from interference of light scattered from inhomogeneities in the refractive index of the material, spaced at scales comparable to optical wavelengths. These materials create vivid structural colors that, by their operational principle, cannot bleach. Structural colored materials are of interest for coloration in future devices and consumables as they can be achieved with biocompatible and environmental friendly materials. ${ }^{[12]}$

Structural colors can arise from ordered Color has a major influence on everyday life, from decisionmaking ${ }^{[1]}$ to general well-being. ${ }^{[2]}$ In nature, colored materials also play an important role in the evolutionary development

Dr. L. Schertel

Department of Chemistry

University of Cambridge

Cambridge CB2 1TN, UK

E-mail: Is849@cam.ac.uk

Dr. L. Schertel, L. Siedentop, Dr. J.-M. Meijer, Dr. P. Keim, Dr. G. J. Aubry,

Prof. G. Maret

Fachbereich Physik

Universität Konstanz

78457 Konstanz, Germany

E-mail: georg.maret@uni-konstanz.de

Dr. L. Schertel, Prof. C. M. Aegerter

Physik-Institut

Universität Zürich

8057 Zürich, Switzerland

Dr. J.-M. Meijer

Institute of Physics

University of Amsterdam

1098 XH Amsterdam, The Netherlands

Dr. G. J. Aubry

Département de Physique

Université de Fribourg

1700 Fribourg, Switzerland

The ORCID identification number(s) for the author(s) of this article can be found under https://doi.org/10.1002/adom.201900442.

DOI: 10.1002/adom.201900442 as well as disordered photonic structures. Periodically ordered photonic structures such as opals where iridescent colors arise from Bragg diffraction are well established. ${ }^{[13-17]}$ Their colors are, however, strongly dependent on the angle of view because of the periodicity of interparticle distance giving rise to narrow diffraction maxima. For certain applications in coatings, paints, as well as cosmetics, this angular dependency can be undesirable. The disordered counterpart of photonic crystals are photonic glasses (PGs), which are random assemblies of monodisperse spherical particles. ${ }^{[18]}$ The isotropic nature of their microscopic structure enables PGs to have angle-independent structural colors, isotropic transport properties, reflectance, transmittance, and photonic (pseudo) band gaps. While these multiple scattering systems were first recognized as improved white paint materials, ${ }^{[19,20]}$ controlled resonant scattering in the visible range ${ }^{[21]}$ allows $P G s$ to produce bright angleindependent structural colors even in relatively low refractive index materials. ${ }^{[10,22]}$

Although the preparation of PGs is relatively well explored, ${ }^{[2,23,24]}$ a limited range of colors was produced so far by self-assembly of monodisperse spheres to PGs. ${ }^{[25-27]}$ Note that more advanced approaches such as core-shell particles and hydrogels produced by microfluidics and nonequilibrium colloidal assembly ${ }^{[28-31]}$ broadened that range of colors but these modifications do come at the expense of scalability of the production process and cost. The lack of a quantitative description of the range of isotropic structural colors in PGs 
has hindered the potential of these low-cost structures for real life applications. Several explanations of structural colors have been proposed so far. For instance, Noh and coworkers have shown the importance of short-range correlations in the structure for the color appearance and proposed a model based on single and double scattering. ${ }^{[32,33]}$ Later Magkiriadou et al. used a quantitative scattering model to explain isotropic structural color in these materials and propose that color appearance is dominated by backscattering resonances of individual particles. ${ }^{[26]}$ They claim that saturated red and yellow cannot be obtained in PGs due to their limited range of reachable hues caused by a blue single particle resonance. Nevertheless, so far the origin of angle-independent structural colors is not fully understood and no corresponding model to show the exact range of accessible colors of PGs has been established.

Here, we show that a recently developed quantitative scattering model ${ }^{[2434]}$ can fully capture the observed isotropic structural colors of PGs produced so far and even predicts a full, repeating color spectrum in PGs. The model takes into account resonant Mie scattering, short-range positional correlations, optical near-field coupling of randomly packed, spherical scatterers as well as the sample's internal reflection at the boundary. The model predicts the reflectance spectra and the expected sample color solely from the scatterer size, refractive index, filling fraction, and sample thickness. We compare the reflectance spectra calculated by the model to spectral reflectance measurements of polystyrene (PS) PGs over the entire visible spectrum. By varying the colloidal scatterer size, we can obtain blue, green, red, and purple colors repeatably, as predicted by the model. In addition, changing the effective sample thickness enables us to tune between vivid colors and white broadband reflectance. The good agreement between the model and experiment for PS PGs enables us to map the parameter space and to predict the range of color hues for different refractive index photonic materials. We show that in higher index PGs, e.g., made of titania, ${ }^{[24]}$ even saturated yellow, magenta, blue, and green can be achieved over four decades of sample thicknesses. Clearly, this model provides a theoretical handle to make use of PGs for structural colors in industrial applications.

As the optical appearance of materials is given by its reflectance spectrum, we calculate the reflectance spectra from the transport mean free path $\ell^{*}$ predicted by the scattering model. $\ell *$ is the length scale over which the memory of the direction of light propagation is lost in the regime of strong multiple light scattering. ${ }^{[24,34]}$ In the situation of high turbidity present in the PGs, the light transport is diffusive on large length scales and $\ell^{*}$ can be seen as the step length in a random walk of photons. $\ell^{*}$ actually quantifies the inverse turbidity and the scattering strength $\lambda / \ell^{*}, \lambda$ being the incident vacuum wavelength. On a scale smaller than $\ell^{*}$, a case sketched in Figure 1a, the light scattering is described by resonant Mie scattering of individual spherical scatterers ${ }^{[35]}$ represented by the form factor $F(\theta)$ ( $\theta$ being the scattering angle) indicated in green. Positional correlations between the scatterers (taken as hard noninterpenetrating spheres) result in interparticle interferences which are accounted for by the Percus-Yevick structure factor $S(\theta),{ }^{[36]}$ sketched in blue. The angulardependent local scattering intensity is $I(\theta)=F(\theta) \cdot S(\theta)$ and $1 / \ell^{*}$ is obtained from angular integration of $I(\theta)$ weighted by $\rho \cdot(1-\cos (\theta)), \rho$ being the scatterers number density. ${ }^{[37]}$ Note that in the evaluation of $I(\theta)$ the choice of an appropriate effective refractive index $n_{\text {eff }}$ of the overall material is crucial. In contrast to earlier introduced models, ${ }^{[26,32,37]}$ the proposed model uses the energy coherent potential approximation (ECPA) for the effective refractive index $n_{\text {eff }}{ }^{[38,39]}$ which automatically accounts for near-field optical coupling between scatterers. For a detailed description of the model, see refs. [24,34], Supporting Information, and Figure S1 in the Supporting Information for the respective contributions of $F(\theta), S(\theta)$, and $n_{\text {eff }}$ to $\ell^{*}$.

We determine the PG reflectance spectra $R(\lambda)$ from $\ell^{*}(\lambda)$ via the transmittance for a slab-shaped sample calculated from the solution of the diffusion equation ${ }^{[40,41]}$

$$
T(\lambda)=\frac{\ell^{*}(\lambda)+z_{0}(\lambda)}{L+2 z_{0}(\lambda)}
$$

a)

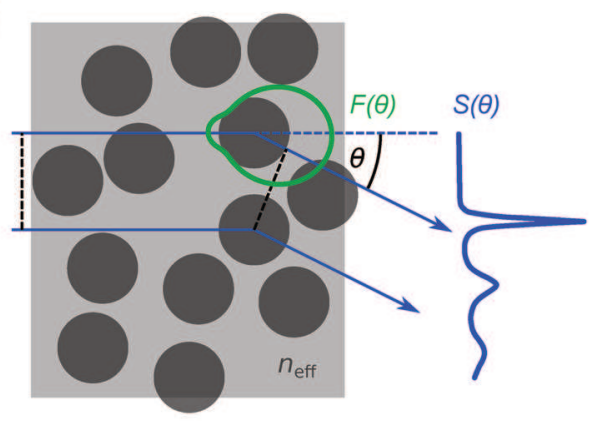

b)

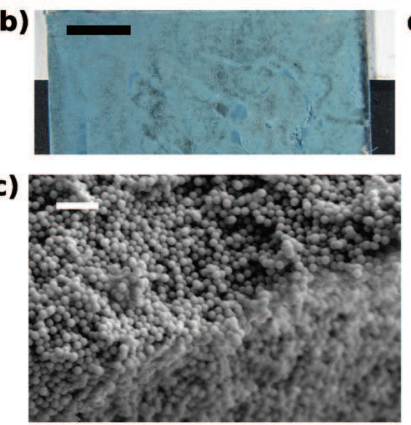

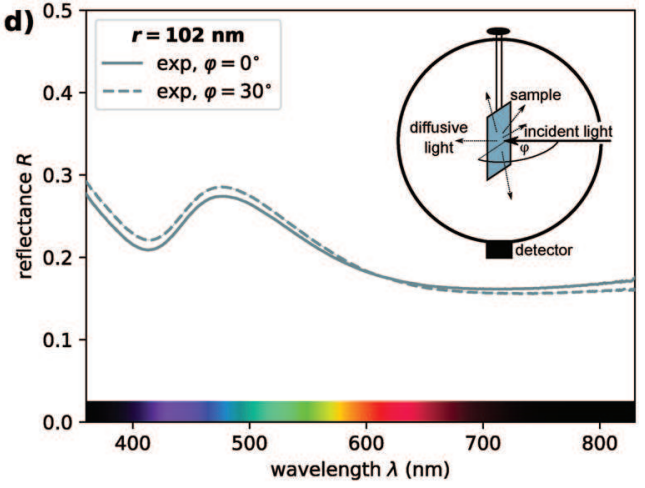

Figure 1. a) Sketch of the different contributions to the scattering in a PG: Mie form factor $F(\theta)$ (green), hard sphere structure factor $S(\theta)$ (blue), and effective refractive index $n_{\text {eff }}$ (gray). b) Photograph of a typical PG consisting of monodisperse PS spheres with radius $r=102 \pm 3 \mathrm{~nm}$ and $0.0125 \mathrm{wt} \%$ CB under diffusive white light illumination showing a blue color (scale bar: $0.5 \mathrm{~cm}$ ). c) SEM micrograph of the sample in (b) showing an edge (top and side view) of the random assembly (scale bar: $1 \mu \mathrm{m}$ ). d) Measured reflectance spectra of the same sample under perpendicular illumination (angle of incidence $\left.\varphi=0^{\circ}\right)$ and under angled illumination $\left(\varphi=30^{\circ}\right)$. Inset in (d): Sketch of the integrating sphere setup. 

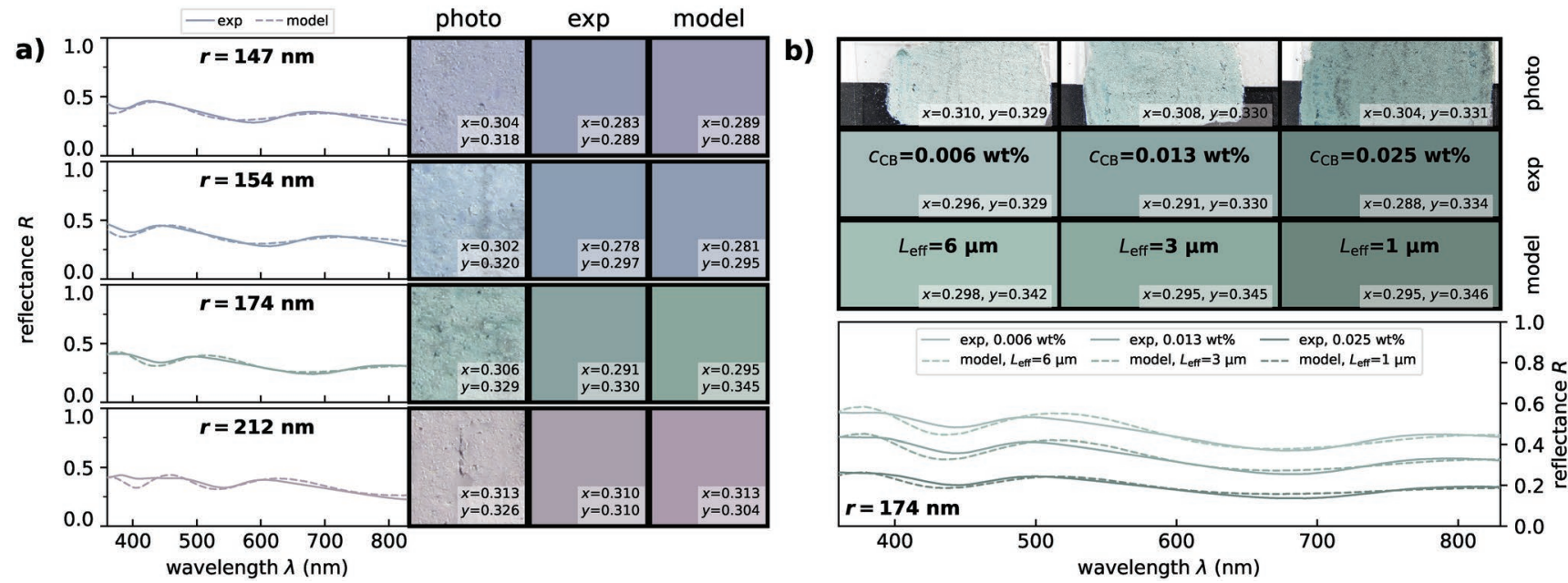

Figure 2. a) Radius sweep: Reflectance spectra of four PG samples with differing particle radius $r$, measured (exp, solid lines) and calculated (model, dashed lines). Photographs (area: $4 \times 4 \mathrm{~mm}^{2}$ ) of the measured samples (photo), color calculated from the measured spectrum (exp), and color calculated by the modeled spectrum (model). b) Effective thickness sweep (top to bottom): Photographs, measured colors, modeled colors, measured and modeled reflectance spectra for three different concentrations of CB $(0.00625,0.0125,0.025 \mathrm{wt} \%)$. The corresponding effective sample thickness $L_{\text {eff }}$ was fitted. White insets show the colors chromaticity coordinates.

with $z_{0}(\lambda)$ being the extrapolation length and $L$ the sample thickness. $z_{0}(\lambda)$ incorporates boundary conditions and internal reflections at the surface of the sample and is calculated via ${ }^{[40]}$

$z_{0}(\lambda)=\frac{2}{3} \ell *(\lambda)\left(\frac{1+R_{\mathrm{i}}}{1-R_{\mathrm{i}}}\right)$

where $R_{\mathrm{i}}$ is the internal reflectivity calculated via the use of Fresnel's law and the Maxwell Garnett effective refractive index. ${ }^{[42]}$ From the transmittance, we finally obtain the reflectance spectrum $R(\lambda)=1-T(\lambda)$.

In Equation (1), the sample thickness $L$ is, besides the particle radius $r$, refractive index and filling fraction, an experimentally accessible parameter. However, the sample thickness is difficult to control on a macroscopic scale in experiments, especially in thin samples as required for isotropic structural colors. A practical means to circumvent a precise thickness control is adding a strong broadband absorber such as carbon black (CB). ${ }^{[10,12]}$ CB lowers the absorption length $L_{\mathrm{a}}$ in the PG sample and thus acts as a cut-off length for the photon path length distribution. Physically, this process eliminates the contributions of long photon paths that generate the white appearance in $R(\lambda)$. Controlling the amount of CB enables the preparation of macroscopically thick samples that still possess bright structural colors. The macroscopic absorption length $L_{\mathrm{a}}$ of such samples acts similar to an effective sample thickness $L_{\text {eff. To }}$ be able to compare the calculated reflectance spectra of the model to experimental data, we replace $L$ by $L_{\text {eff }}$ in Equation (1) and use it as a fitting parameter. Note that this is an approximation as the photon path length distribution is somewhat different for a nonabsorbing sample of finite thickness $L$ and a sample with a corresponding absorption length $L_{\mathrm{a}}$. Note further that Equation (1) is fully valid only in the diffusive limit $L \gg \ell^{*}$, but may still be used here as a simple approximation for the calculation of $R$, as fitting the effective sample thickness $L_{\text {eff }}$ in the model essentially adjusts the amplitude of the reflectivity while its spectral dependence originates from the wavelength dependence of $\ell *$.

Having outlined the model calculation, we now show a typical experimental PG. Figure $1 \mathrm{~b}$ shows a photograph of a PG sample, where a blue structural color can be observed. We prepared the PG sample of PS spheres with $r=102 \pm 3 \mathrm{~nm}$ on a microscope slide according to the method of Magkiriadou et al. ${ }^{[2]}$ (For details, see the Experimental Section and Figure S2, Supporting Information.) A total concentration of $0.0125 \mathrm{wt} \% \mathrm{CB}$ is present in the PG sample, which reduces the optical path length, as explained above. Figure 1c shows a typical scanning electron microscope (SEM) micrograph of the same sample, where the amorphous and random assembly of the spheres is visible. The reflectance spectra of the sample were measured as a function of wavelength using a UV-Vis spectrometer with an integrating sphere as detector unit under vertical illumination (angle of incidence $\left.\varphi=0^{\circ}\right)$ and angular illumination $\left(\varphi=30^{\circ}\right)$. The normalized spectra for both angles of incidence are shown in Figure 1d, where the inset shows a schematic of the integrating sphere setup. The clear similarity between both curves demonstrates the optical isotropy while the peak around $480 \mathrm{~nm}$ is responsible for the blue structural color.

In the following section, we quantitatively compare our model to experimental spectra and explore the effect of scatterer size and sample thickness. A series of 13 samples with particle sizes varying from $r=59 \mathrm{~nm}$ to $r=251 \mathrm{~nm}$ was prepared as described above. Figure 2a shows four representative PG samples consisting of PS spheres with $r=147,154,174$, and $212 \mathrm{~nm}$, respectively.

A change of the particle radius $r$ of less than $100 \mathrm{~nm}$ already leads to a dramatic change of the observed isotropic structural color hues. A crossover from purple to blue via green to dark red is observed in the photographs in Figure 2a. 
The full series of particle sizes is shown in Figure S3 in the Supporting Information where even a repeating color sequence is observed. We measured reflectance spectra (solid lines) of each sample and compared them to the proposed model (dashed lines). In the model, the experimentally determined radii are used without fitting, although a polydispersity of $5 \%$ is employed in the model to account for the small experimental polydispersity. In addition, a filling fraction of $f=0.3$ is used which is similar to the earlier experimentally verified value for dried PGs. ${ }^{[24]}$ Note that this filling fraction is relatively low compared to the sediment value obtained directly after centrifugation, but slipcasting and drying leads to a network like packing rather than to a dense particle packing. The only fitting parameter is the sample thickness $L_{\text {eff }}$ that is determined via a least-squares fit. Very good agreement of the position of the observed resonances between the model and experimental curves is found. For the smallest particle size $(r=93 \mathrm{~nm})$, only the tail of the first resonance is observed in the low wavelength region, resulting in the dark blue color. The blue line in Figure S1 in the Supporting Information shows the relevance of structural correlations for the position of the resonances. The first resonance is affected by structural correlations but the higher resonances are essentially not. With increasing $r$ the resonance shifts from blue $(r=93 \mathrm{~nm})$ to green $(r=114 \mathrm{~nm})$ to red $(r=128 \mathrm{~nm})$. A second resonance appears in the low wavelength region of the spectrum of the sample with $r=147 \mathrm{~nm}$ and explains its purple appearance. The shift of both resonances with further increasing particle size leads to the repeating color scheme observed in Figure 2a. The appearance of a second resonance with increasing $r$ also explains earlier observations of blue dominated structural color in PGs. ${ }^{[26]}$ However, our results show that this resonance does not eliminate intermediate colors like green and red but is very sensitive to a small change in $r$.

Note that for samples with larger particle radii, the pure color appearance smears out as multiple resonances are present in the visible range of the spectrum (see also Figure S3, Supporting Information). These higher-order resonances are further strongly affected by small polydispersity in the experimental data and thus show small deviations from the model predictions for large particle sizes. Note that the scattering behavior in this regime was previously studied ${ }^{[20]}$ and fully explained recently. ${ }^{[34,43]}$

Besides the photographs, Figure 2a also shows the experimental and calculated spectra $R(\lambda)$ converted into colors as perceived by the human eye. The color sensation is approximated via the open-source python module colorpy, ${ }^{[44]}$ which allows to convert any spectrum in the visible range (we use the range $\lambda=[360,830] \mathrm{nm}$ ) into a color space such as Commission internationale de l'éclairage (CIE) $x y Y$ or Red Green Blue, making the color printable or displayable on a screen. See Figure S4 in the Supporting Information and, e.g., ref. [45] for the derivation of the color spaces and the $x y$-chromaticity coordinates. By comparing the chromaticity coordinates between the measured and the modeled spectrum, we see a remarkably good agreement between the colors of the measured and modeled spectrum as well as with the colors observed in the photographs.
The second parameter we investigated is the effect of absorption as controlled by the amount of $\mathrm{CB}$ which is equivalent to changing the effective sample thickness $L_{\text {eff }} \cdot{ }^{[10,12]}$ We prepared three samples with well-defined concentrations of CB $(0.00625$, $0.0125,0.025 \mathrm{wt} \%)$. Photographs of these samples are shown in Figure $2 \mathrm{~b}$. Decreasing the CB concentration increases the effective sample thickness $L_{\text {eff }}$, changes the color saturation, and generally leads to a lighter color appearance. Again, the model's $L_{\text {eff }}$ value was fitted in the same manner to the experimental data. With increasing effective sample thickness, multiple scattering contributions become more relevant and the appearance becomes more whitish as the resonance amplitudes become smaller in respect to the mean reflectance. A crossover from vivid colors to white broadband reflectance can be seen with increasing sample effective thickness. The hue can be quantified by the chromaticity coordinates which are given in each color patch. We note that the effective thickness does not alter the hue significantly.

We are now able to use the proposed model and color conversion method to map the full relevant parameter space of isotropic structural colors for a specific refractive index of the particles. In Figure 3a, we plot a map of colors calculated for PS PGs $(n=1.6)$ by the model, scanning the sample thickness and the particle radius. A filling fraction of $f=0.3$ and a polydispersity of $5 \%$ was used as in the experiments. The corresponding colors of all the experimentally prepared PGs are indicated in the map as blue dots (Figure 2) or black crosses (Figure S3, Supporting Information).

The color change with increasing particle size observed in Figure 2 is clearly visible in the maps. Note that a pure blue is only possible for very small particle sizes $r<95 \mathrm{~nm}$ in the crossover from Rayleigh to Mie-scattering and additionally for $r \approx 150 \mathrm{~nm}$. Changing the sample thickness (note the logarithmic scale) changes mainly the color saturation. The most intense colors of PS PG appear for particle radii between 50 and $300 \mathrm{~nm}$ and an effective sample thicknesses from 0.5 to $30 \mu \mathrm{m}$.

A prominent ingredient in white paints and other strongly multiple scattering materials is titanium dioxide $\left(\mathrm{TiO}_{2}\right)$ because of its high refractive index combined with low absorption in the visible. Strong resonant behavior was observed and understood with the ECPA scattering model in PGs of $\mathrm{TiO}_{2}$ colloids. ${ }^{[24]}$ In Figure 3b, we show a map of PGs of perfectly spherical and monodisperse particles of $n=2.0$ (amorphous $\mathrm{TiO}_{2}$ ) and a filling fraction of $f=0.5$. The higher index leads to more saturated colors due to the enhanced resonant scattering in these materials. We observe that for particle sizes of 100-150 nm strongly saturated yellow, magenta, cyan, and green can be achieved over several orders of magnitude of sample thickness. The predicted saturated yellow and magenta in the $\mathrm{TiO}_{2}$ map are in contrast to ref. [26], where an absence of saturated red and yellow was claimed in PGs.

Further quantification is given by calculating the points in CIE $x y$ space that are possible to reach with a PG. We call this the gamut of the PG. The gamuts for PS PGs and $\mathrm{TiO}_{2} \mathrm{PGs}$ are shown in Figure $3 c$, respectively, $d$. The gamut is made up out of loops (each corresponding to an effective thickness sweep at fixed particle radius) that contain the white point W: these are the high and low limits of the effective sample 

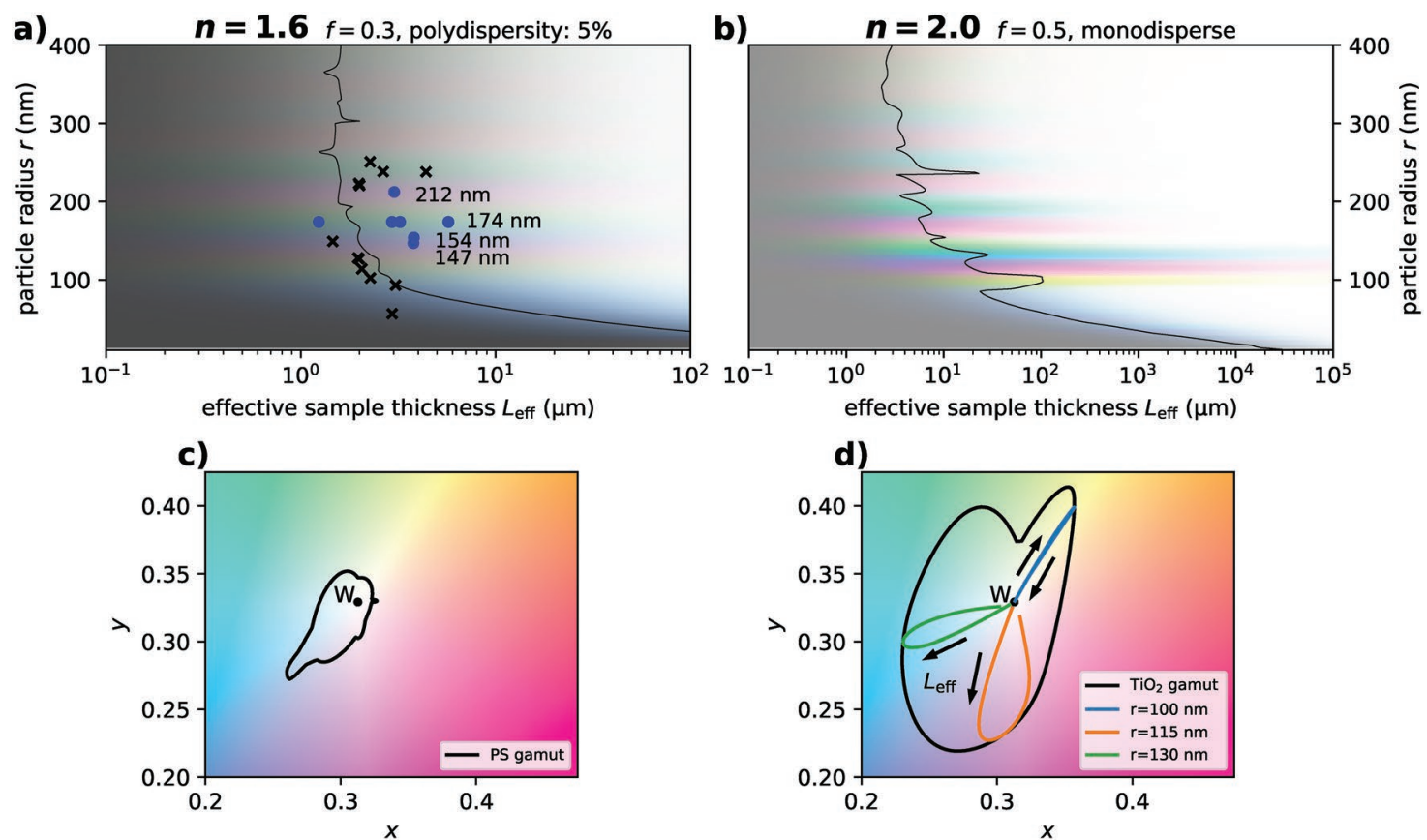

Figure 3. a) Color map for varying radius and sample thickness for a PG with refractive index $n=1.6$, filling fraction $f=0.3$, and a particle polydispersity of 5\%. Blue dots correspond to the measured samples from Figure 2. Black crosses correspond to samples of Figure S3 in the Supporting Information. The black line indicates the sample thickness $L$ that gives maximum color saturation for a corresponding particle radius $r$. b) Color map for a PG with refractive index $n=2.0$, filling fraction $f=0.5$, and no particle polydispersity. The black line indicates the maximum saturation as in (a). Panels (c,d) show the gamut reachable for PS PGs and $\mathrm{TiO}_{2} \mathrm{PGs}$, respectively, i.e., the colors shown in (a) and (b), respectively. The three loops shown in (d) correspond to a CB concentration sweep for three different radii. The arrows denote direction of increasing $L_{\text {eff. }}$

thickness, where the sample is white and black, respectively, with vanishing color saturation. Three exemplary loops are shown in Figure 3d. Changing the particle radius rotates the loop around the white point. Note that these loops are very narrow for low refractive indices, i.e., $L_{\text {eff }}$ solely determines the color saturation and $r$ solely determines the color hue. There exists a sample thickness $L$ for which the color is the most saturated for each $r$, i.e., the distance farthest away from the white point. These $L_{\text {eff }}$-values of maximum saturation are shown in Figure 3a,b as black lines. Note that our experimentally prepared PS samples are very close to these values. We observe that the point of maximum saturation for each loop does not make up the boundary of the gamut. This can especially be seen for $r=115 \mathrm{~nm}$ in Figure 3d. To obtain the gamuts boundary, we calculate the CIE $x y$-values for a tightly sampled parameter space and calculated the concave hull via the $k$-nearest neighbor approach. ${ }^{[46]}$ In terms of area of the complete visible gamut, we obtain $0.68 \%$ for the PS PGs gamut and $4.28 \%$ for the $\mathrm{TiO}_{2}$ PGs gamut.

The asymmetry of the reachable area of colors around the white point in Figure $3 \mathrm{c}, \mathrm{d}$ shows the difficulty to obtain red and yellow. Note that for PS PGs, especially saturated blue is easily achievable (peak in the area to lower left corner). This might explain the increased occurrence of blue in natural structures such as bird feathers and beetles. ${ }^{[10,26]}$

In conclusion, we showed that even with low refractive index material (PS), blue, green, and red isotropic structural colors from PGs can be prepared for a wide range of parameters. This might enable fabrication of biocompatible and sustainable paints and coatings by replacing current scatterer's materials with an environmental friendly material such as cellulose. ${ }^{[47,48]}$ All our data are explained by a recently proposed model. ${ }^{[24,34]}$ The very good agreement of the predicted and observed isotropic structural colors allows us to use the model to predict the reachable color space for other refractive index materials. We show that saturated green, cyan, yellow, and magenta can be reached in higher index PGs. ${ }^{[24]}$ Other approaches such as coated spheres were used earlier to tune the contribution of structural correlations and Mie-scattering separately. ${ }^{[28]}$ This allows to reach saturated colors in even lower index materials. Replacing the spherical bulk scatterers in the model by coated spheres would allow to predict colors even in these materials. Similarly, it was recognized that photonic structures found in nature often uses inverse structures, ${ }^{[10]}$ which is intrinsically included in the proposed scattering model.

\section{Experimental Section}

Sample Preparation: PS spheres with different sizes $(r=59 \mathrm{~nm}$ to $r=$ $251 \mathrm{~nm}$ ) were obtained from microparticles $\mathrm{GmbH}$. All PS spheres were characterized by transmission electron microscopy (Zeiss Libra120). For each particle, its size was determined for at least 100 particles. For more details see Supporting Information.

PGs were prepared according to the method of Magkiriadou et al.[26] Typically, $800 \mu \mathrm{L}$ of $5 \mathrm{wt} \%$ dispersion of monodisperse PS spheres in water was mixed with $50 \mu \mathrm{L}$ aqueous dispersion containing 1 wt\% CB (Cabot VXC72R) and 2 wt\% Pluronic F-127 (Sigma-Aldrich). The Pluronic provided stabilization and kept the CB particles suspended. 
Next, the mixture was centrifuged at $10000 \mathrm{rpm}$ for $30 \mathrm{~min}$ and the supernatant was removed, thereby reaching a typical filling fraction of $f \approx 0.47 \pm 0.05$. The dense dispersion was slipcasted onto a microscope slide and the water was allowed to evaporate, resulting in an amorphous assembly of the spheres with a thickness of several hundred $\mu \mathrm{m}$.

Sample Characterization: The structure of the particle assemblies was characterized in detail with SEM (Zeiss CrossBeam 1540XB). Samples were broken to reveal the inner structure of the PGs.

Color photographs of the PG samples on a white and black background were captured with a digital camera (Canon PowerShot SX30 IS, settings: $1 / 40 \mathrm{~s}$ exposure time, aperture F2.7, ISO 80, manual focus: $4.3 \mathrm{~mm}$ focal length) under illumination of white diffusive light from a halogen lamp (Intralux DC 1100 and Fiber-Lite PL-800). The images were normalized using the custom white balance of the camera to a white background (Figure 1b).

Reflectance spectra of the PG samples were measured in a UV-Vis spectrometer with an integrating sphere as detector unit (Agilent Cary 5000). Spectra were measured from 360 to $830 \mathrm{~nm}$ in $1 \mathrm{~nm}$ steps and with a spectral bandwidth (FWHM) of $2 \mathrm{~nm}$. The circular illuminating beam had a diameter of $\approx 0.5 \mathrm{~cm}$. A reference beam was used for calibration and a background (measurement without sample) was subtracted from the data. As the samples were relatively thick and contained $C B$, a strong broadband absorbing material, the remaining transmission could be assumed to be zero.

\section{Supporting Information}

Supporting Information is available from the Wiley Online Library or from the author.

\section{Acknowledgements}

L.S. and L.S. contributed equally to this work. The authors thank Prof. Schmidt-Mende for providing the Cary 5000 Spectrometer. The authors also acknowledge support by the Deutsche Forschungsgemeinschaft in the frame of SFB1214 project B2 and the Swiss National Science Foundation under project 200020M_162846. J.-M.M. acknowledges financial support from the Alexander von Humboldt Foundation.

\section{Conflict of Interest}

The authors declare no conflict of interest.

\section{Keywords}

color modeling, disordered photonics, gamut, isotropic structural colors, monodisperse colloidal glasses

Received: March 12, 2019

Revised: April 25, 2019

Published online:

[1] A. Bechara, H. Damasio, A. R. Damasio, Cereb. Cortex 2000, 10, 295.

[2] K. Yildirim, A. Akalin-Baskaya, M. Hidayetoglu, Build. Sci. 2007, 42, 3233.

[3] M. J. Ryan, Science 1998, 281, 1999.

[4] S. M. Doucet, M. G. Meadows, J. R. Soc., Interface 2009, 6, S115.
[5] B. D. Wilts, K. Michielsen, H. De Raedt, D. G. Stavenga, Proc. Natl. Acad. Sci. USA 2014, 111, 4363.

[6] B. D. Wilts, X. Sheng, M. Holler, A. Diaz, M. Guizar-Sicairos, J. Raabe, R. Hoppe, S.-H. Liu, R. Langford, O. D. Onelli, D. Chen, S. Torquato, U. Steiner, C. G. Schroer, S. Vignolini, A. Sepe, Adv. Mater. 2018, 30, 1702057.

[7] P. Vukusic, J. R. Sambles, Nature 2003, 424, 852.

[8] R. O. Prum, R. H. Torres, S. Williamson, J. Dyck, Nature 1998, $396,28$.

[9] P. Vukusic, J. R. Sambles, C. R. Lawrence, R. J. Wootton, Proc. R. Soc. London, Ser. B 1999, 266, 1403.

[10] J. D. Forster, H. Noh, S. F. Liew, V. Saranathan, C. F. Schreck, L. Yang, J.-G. Park, R. O. Prum, S. G. J. Mochrie, C. S. O'Hern, H. Cao, E. R. Dufresne, Adv. Mater. 2010, 22, 2939.

[11] E. S. A. Goerlitzer, R. N. K. Taylor, N. Vogel, Adv. Mater. 2018, 30, 1706654.

[12] Y. Takeoka, Chem. Commun. 2018, 54, 4905.

[13] E. Yablonovitch, Phys. Rev. Lett. 1987, 58, 2059.

[14] E. Yablonovitch, T. J. Gmitter, Phys. Rev. Lett. 1989, 63, 1950.

[15] C. López, Adv. Mater. 2003, 15, 1679.

[16] G. von Freymann, V. Kitaev, B. V. Lotsch, G. A. Ozin, Chem. Soc. Rev. 2013, 42, 2528.

[17] S. Kinoshita, S. Yoshioka, J. Miyazaki, Rep. Prog. Phys. 2008, 71, 076401.

[18] J. Ballato, J. Opt. Soc. Am. B 2000, 17, 219.

[19] P. D. García, R. Sapienza, A. Blanco, C. López, Adv. Mater. 2007, 19, 2597.

[20] P. D. García, R. Sapienza, C. López, Adv. Mater. 2010, 22, 12.

[21] R. Sapienza, P. D. García, J. Bertolotti, M. D. Martín, A. Blanco, L. Vina, C. López, D. S. Wiersma, Phys. Rev. Lett. 2007, 99, 233902.

[22] B. Q. Dong, X. H. Liu, T. R. Zhan, L. P. Jiang, H. W. Yin, F. Liu, J. Zi, Opt. Express 2010, 18, 14430.

[23] M. Chen, D. Fischli, L. Schertel, G. J. Aubry, B. Häusele, S. Polarz, G. Maret, H. Cölfen, Small 2017, 13, 1701392.

[24] L. Schertel, I. Wimmer, P. Besirske, C. M. Aegerter, G. Maret, S. Polarz, G. J. Aubry, Phys. Rev. Mater. 2019, 3, 015203.

[25] Y. Takeoka, S. Yoshioka, A. Takano, S. Arai, K. Nueangnoraj, H. Nishihara, M. Teshima, Y. Ohtsuka, T. Seki, Angew. Chem., Int. Ed. 2013, 52, 7261.

[26] S. Magkiriadou, J.-G. Park, Y.-S. Kim, V. N. Manoharan, Phys. Rev. E 2014, 90, 062302.

[27] H. Galinski, G. Favraud, H. Dong, J. S. T. Gongora, G. Favaro, M. Döbeli, R. Spolenak, A. Fratalocchi, F. Capasso, Light: Sci. Appl. 2017, 6, e16233.

[28] J.-G. Park, S.-H. Kim, S. Magkiriadou, T. M. Choi, Y.-S. Kim, V. N. Manoharan, Angew. Chem., Int. Ed. 2014, 53, 2899.

[29] J.-G. Park, W. B. Rogers, S. Magiriadou, T. Kodger, S.-H. Kim, Y.-S. Kim, V. N. Manoharan, Opt. Mater. Express 2017, 7, 253.

[30] L. Bai, V. C. Mai, Y. Lim, S. Hou, H. Möhwald, H. Duan, Adv. Mater. 2018, 30, 1705667.

[31] G. Shang, L. Maiwald, H. Renner, D. Jalas, M. Dosta, S. Heinrich, A. Petrov, M. Eich, Sci. Rep. 2018, 8, 7804.

[32] H. Noh, S. F. Liew, V. Saranathan, R. O. Prum, S. G. J. Mochrie, E. R. Dufresne, H. Cao, Phys. Rev. E 2010, 81, 051923.

[33] H. Noh, S. F. Liew, V. Saranathan, S. G. J. Mochrie, R. O. Prum, E. R. Dufresne, H. Cao, Adv. Mater. 2010, 22, 2871.

[34] G. J. Aubry, L. Schertel, M. Chen, H. Weyer, C. M. Aegerter, S. Polarz, H. Cölfen, G. Maret, Phys. Rev. A 2017, 96, 043871.

[35] C. F. Bohren, D. R. Huffman, Absorption and Scattering of Light by Small Particles, Wiley, New York 1998.

[36] J. K. Percus, G. J. Yevick, Phys. Rev. 1958, 110, 1.

[37] S. Fraden, G. Maret, Phys. Rev. Lett. 1990, 65, 512.

[38] K. Busch, C. M. Soukoulis, Phys. Rev. Lett. 1995, 75, 3442.

[39] K. Busch, C. M. Soukoulis, Phys. Rev. B 1996, 54, 893. 
[40] N. Garcia, A. Z. Genack, A. A. Lisyansky, Phys. Rev. B 1992, 46, 14475.

[41] M. Reufer, L. F. Rojas-Ochoa, S. Eiden, J. J. Sáenz, F. Scheffold, Appl. Phys. Lett. 2007, 91, 171904.

[42] J. X. Zhu, D. J. Pine, D. A. Weitz, Phys. Rev. A 1991, 44, 3948.

[43] D. Montesdeoca, F. Bayat, A. Espinha, A. Blanco, C. Pecharromán, C. López, Part. Part. Syst. Charact. 2016, 33, 871.

[44] M. Kness, Colorpy: A Python Package for Handling Physical Descriptions of Color and Light Spectra, https://github.com/markkness/ColorPy (accessed: January 2019).
[45] D. Brainard, A. Stockman, in Handbook of Optics (Volume III): Vision and Vision Optics (Eds: M. Brass, J. Enoch, E. Van Stryland, W. Wolfe), McGraw-Hill Education, New York 2010, Ch. 10.

[46] A. Moreira, M. Yasmina Santos, in Proc. Second Int. Conf. Computer Graphics Theory and Applications (GRAPP 2007), Vol. 1 (Eds.: J. Braz, P.-P. Vázquez, J. M. Pereira), INSTICC, SciTePress, Sebutal, Portugal 2007, pp. 61-68.

[47] J. Zhang, T. J. Elder, Y. Pu, A. J. Ragauskas, Carbohydr. Polym. 2007, 69, 607.

[48] C.-F. Yan, H.-Y. Yu, J.-M. Yao, Cellulose 2015, 22, 3773. 